\title{
Deep Learning based Brain Tumour Segmentation
}

\author{
PATTABIRAMAN V. \\ HARSHIT SINGH \\ Vellore Institute of Technology - Chennai Campus Vellore Institute of Technology - Chennai Campus \\ Computer Science and Engineering \\ Chennai, Tamil Nadu \\ INDIA \\ Computer Science and Engineering \\ Chennai, Tamil Nadu \\ INDIA \\ pattabiraman.v@vit.ac.in \\ harshitrbsingh@gmail.com
}

\begin{abstract}
Artificial Intelligence has changed our outlook towards the whole world and it is regularly used to better understand all the data and information that surrounds us in our everyday lives. One such application of Artificial Intelligence in real world scenarios is extraction of data from various images and interpreting it in different ways. This includes applications like object detection, image segmentation, image restoration, etc. While every technique has its own area of application image segmentation has a variety of applications extending from complex medical field to regular pattern identification. The aim of this paper is to research about several FCNN based Semantic Segmentation techniques to develop a deep learning model that is able to segment tumours in brain MRI images to a high degree of precision and accuracy. The aim is to try several different architecture and experiment with several loss functions to improve the accuracy of our model and obtain the best model for our classification including newer loss function like dice loss function, hierarchical dice loss function cross entropy, etc.
\end{abstract}

Key-Words: - FCNN, MRI, Dice Loss, ReLU, Leaky ReLU, U-Net, Segmentation.

Received: May 25, 2020. Revised: December 3, 2020. Accepted: December 19, 2020. Published: December 29, 2020.

\section{Introduction}

The rate of brain tumour cases among all the age groups is increasing at an alarming rate all over the world. Gliomas [1] are the most common form of brain tumours found but they are comparatively low grade i.e the life expectancy of the patient suffering for glioma is several years. On the other hand, more aggressive tumours reduce the life expectancy of the patient to a maximum of two years.

In the earlier stages of the cancer, growth of the tumour can be slowed down using containment measures like chemotherapy, radiation, etc. MRI scans give the optical images of the brain which help the doctors to identify these tumours and these images also enable the doctors to predict the growth of the tumour at early stages and help to reduce the growth and impact of the tumour on the patient's life. Different tumours like gliomas or glioblastomas or meningiomas all present different levels of difficulties in identification like gliomas and glioblastomas are much more difficult to localize. Such tumours (along with the neighbouring edema) have diffused and poorly contrasted shapes and properties and exhibit structures like tentacles that make them difficult to segment [2].
Malignant brain tumours are very tricky to detect and they evade the eyes of specialists too in some cases. Also, with malignant tumours there is always a risk that these tumour cells can start corroding and affecting the nearby healthy and normal cells. for patients to get second opinion from tumour experts all around the globe.

Brain tumour segmentation consists of extracting the tumour region from healthy brain tissues; the existence of brain tumours can often be detectable. However, accurate and effective segmentation of tumours remains a challenging task, since the tumours can occur in different regions of the brain and their shapes and sizes are almost always different. These tumours are very complex in shape and do not exhibit any rigid shape or size and they have very different appearance properties.

\section{Literature Survey and Existing Model}

Artificial Intelligence has changed our outlook towards the whole world. AI enables us to find hidden features and relation between objects that are overlooked by the common eye. E.g a human brain cannot calculate the dependence of a particular result 
based on thousands of attributes. We cannot take into account all this huge amount of data and this is where AI comes in and helps us solve the problem of dealing with huge dataset and processing them to better understand and interpret them.

AI has been applied in various sectors in the medical field some of them including managing of health records, creation of new drugs, analysing large amounts of reports to get a consensus about the general health in the particular area, etc. AI has also been deployed in segmenting MRI scans of various organs to effectively segment the boundary lines of the organs or the anomalies in the human body. As more and more data is acquired via the internet the accuracy of these models have been increasing everyday encouraging more and more studies and researches are conducted in this specific area of Artificial Intelligence [3].

Segmenting these MRI images has been going on for a long time now and we are now able to see effective results owing to the previous efforts made by the researchers to make image segmentation more and more effective. Various segmentation techniques including clustering based, edge detection based or region based segmentation have been tried out to gauge their accuracy in segmenting all the images presented to them to a high degree of precision.

Menze et al. [4] notes that more and more publication are diverting their primal attention towards brain tumour segmentation. In fact, the number of research paper on this subject have grown exponentially over the last few decades. Thus, highlighting that this area is one of the most sought field of study in the AI and signifying that the need for Deep learning based segmentation tools.

Brain tumour segmentation models can be mainly categorized into two different types - Generative models and Discriminative models. The success of generative models depends majorly on initial domain - specific awareness on the subject of looks and properties of the tumour and healthy tissues. Tissues are very hard to label and segregate and generally present models identify a tumour by distinguishing a tumour from other cells based on shape or signals from the normal brain.

Newer approaches don't take into account prior knowledge or the brain's normal structure and solely depend on extracting low level features thereby creating a model between these identified features and the labels of the voxel. Moreover, discriminative models traditionally use AI pipeline depending close by planned highlights. In order for the success of such strategies, the classifier is developed to distinguish noise from semi-fluid tissues with the expectation that information highlights have an adequately high distinguishing force since the properties the classifying technique is independent from properties of those highlights.

Dong et al., [5] put forward non-invasive magnetic resonance techniques as an identifying tool for identifying tumours in the brain without the risk associated with ionizing radiation. The success of hand drawn segmentation in 3 dimensional images relies heavily on the skill of the operator and takes a lot of time. In order to overcome these backlogs UNet architecture was suggested by the author.

Cui et al., [6] using cascaded deep learning convolutional neural network made an autonomous segmentation model. It consists of two types of networks; tumour localization network (TLN) and a intra tumour classification network (ITCN). TLN helps to separate the tumour region from the MRI images and ITCN helps to segregate the tumour region into more smaller regions.

Chinmayi et al., [7] using the Bhattacharya coefficient, developed a model for Tumour segmentation and classification. An anisotropic filter is used to remove the unwanted regions in the MRI like the skull. It uses deep learning CNN to train the MRI brain tumour image. Further, it utilizes a fastbounding box algorithm to extract the tumour area.

Advantages and Disadvantages of the studied research papers:

The Dong et al. [5] model was able to complete the cross-validation session faster than previous models approximately 30 seconds. The model displayed high efficiency in segmenting tumour images in the BRATS dataset. As compared to previous works they were able to get better DSC in segmentation. Some limitations include the use of cross validation technique which can result in different results in different datasets. Also, parameter tuning was done based on previously conducted empirical study.

The Cui et al.[6] model was able to produce better DSC values compared to the Dong et al.[5] model in overall tumour cases rather than just core tumour cases. Also, this model had better values in the enhancing tumour category and core tumour category. The computation time was also better as 
compared to Dong et al., [5] model due to the employment of 2 distinct FCN models and 1 of them needs only forward computation. This model's accuracy can be improved in core tumour category.

The Chinmayi et al., [7] model was trained on datasets downloaded from Harvard Medical School, Zanjan MRI centre, OASIS dataset and the ADNI dataset. The bounding box algorithm yielded better results than previous works and had an accuracy of 98 percent.

\section{System Implementation}

\subsection{Dataset}

Our model was trained on the LGG MRI dataset as it had more images and class imbalance was less compared to the BRATS dataset. LGG MRI dataset comprises of MRI scan images along with FLAIR abnormality masks.

The images are released by an organisation called the The Cancer Imaging Archive(TCIA). These images are the MRI scans of 110 patients who belonged to The Cancer Genome Atlas (TCGA).

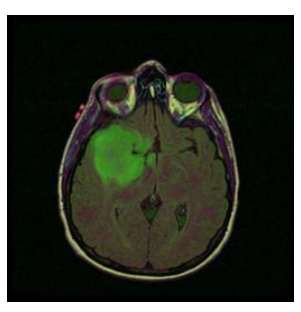

Figure 5.1.1 - Sample Image

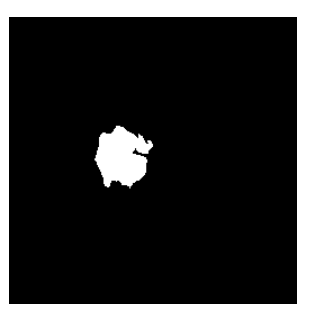

Figure 5.1.2 - The manual marking of tumour
Figure 1: Sample Dataset images

\subsection{Methodology}

The development of the model was done in three phases:

1. Pre-processing the images

2. Segmenting the images

\section{(Phase 1) Pre Processing the images:-}

The images in the dataset were not homogenous and they varied in terms of their shapes and sizes. Hence all these images had to be made of the same size and the dataset was resampled by using the following steps:

- All the images were scaled to the same shapes and sizes to make a common frame of reference
- Unnecessary or noise features like the outlining of the skull was removed to make our model more efficient.

- $\quad$ The dataset was normalized.

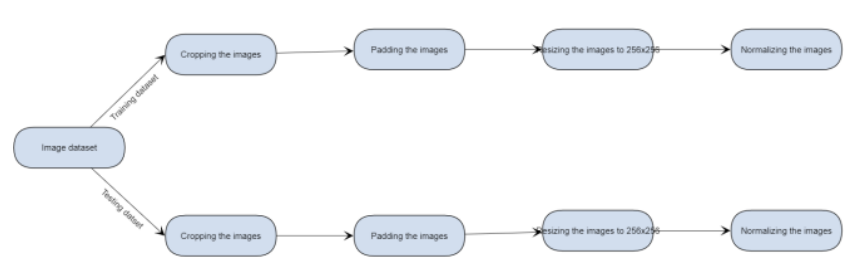

Figure 2: Pre-processing and validation steps

\section{(Phase 2) Segmenting the images: -}

U-Net architecture implemented with convolutional Neural networks was used for segmentation.

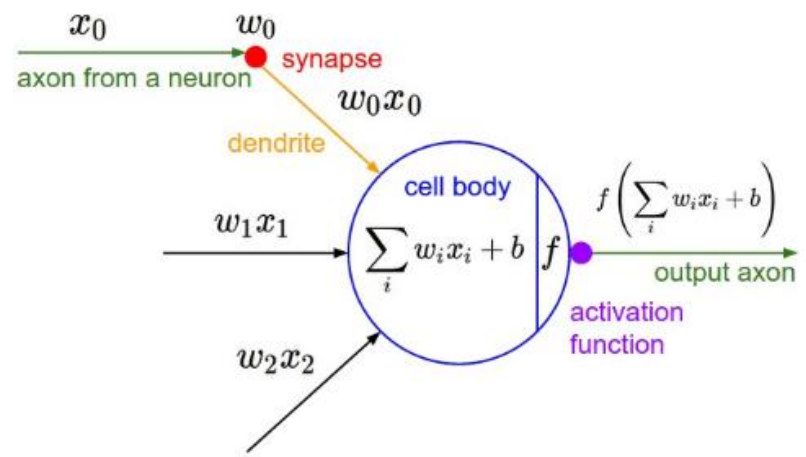

Figure 3: Mathematical model of a Neuron in a Neural Network

ReLU and Leaky ReLU activation functions:

Mathematical equation of the ReLU activation function:

$\mathrm{F}(\mathrm{z})=\max (0, \mathrm{z}) \ldots \ldots \ldots$

Here output of the activation function $F(z)$ gives and output of 0 when the value of $z$ is negative and gives the output as $\mathrm{Z}$ when the number $\mathrm{z}$ is greater than 0 .

Hence, we see that the range of this ReLU activation function is from 0 to infinity. 


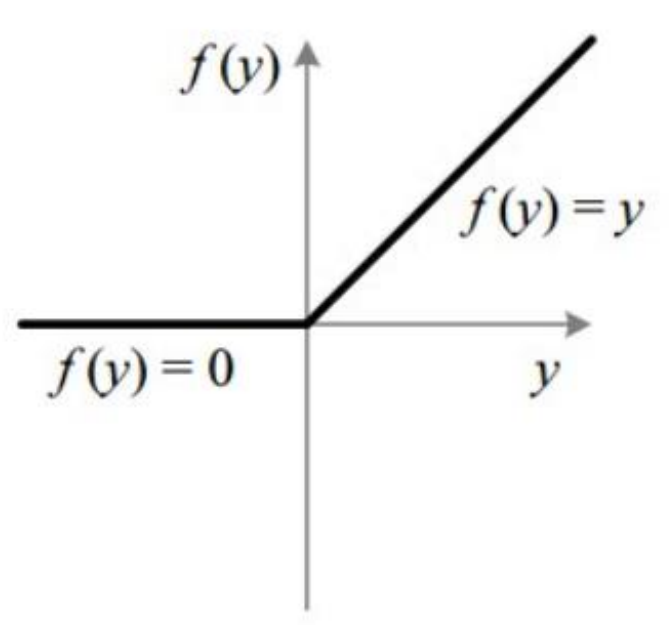

Figure 4: Graphical representation of the ReLU function

The problem with ReLU function is that when negative values get passed to our function , ReLU converts them automatically to 0 and thus the negative values are not marked appropriately thus decreasing the accuracy of our model.

Leaky ReLU activation function:

In order to overcome the shortcomings of ReLU function of not marking the negative values, Leaky ReLU activation function was proposed.

$\mathrm{F}(\mathrm{z})=\{\mathrm{az}, \mathrm{z}<0$

$$
\mathrm{Z}, \mathrm{z}>0\} \ldots \ldots
$$

Here, a is a constant which mostly takes the value of 0.1 .

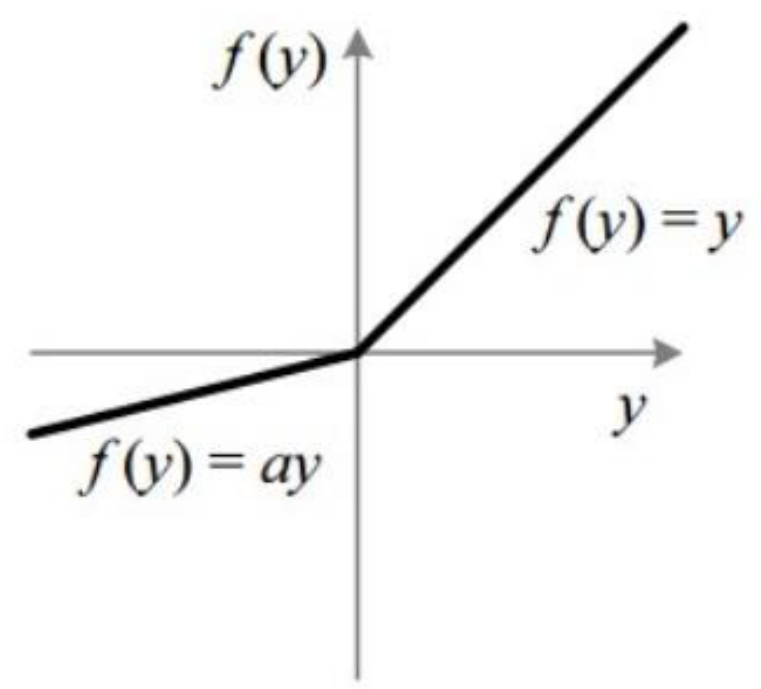

Figure 5: Leaky ReLU function

So, we see that Leaky ReLU function can correctly plot and guage the negative values in the equation thus increasing the accuracy of our model.

Activation function used in this U-Net architecture:

Leaky ReLU (Leaky Rectified Linear Unit)

Leaky ReLU provides better results in many cases compared to other activation functions like ReLU, ELU(Exponential Linear Unit) and GeLU(Gaussian Error Linear Unit).

Thus, after conducting this literature survey, we decided to apply the U-Net architecture with Leaky ReLU activation function and have a max pooling layer after each layer.

\section{Architecture of Our model}

Our architecture has 4 levels of 2 neural network layers with Leaky ReLU activation function and a max pooling layer. In the left side of the $U$ the images are defragment in the successive layers and in the right side the images are constructed back in a bottom to top manner.

The images in the dataset have manual markings of the tumours identified in the MRI scans and these manual markings are used to check the precision and accuracy of our model.

The images in our dataset were sliced and the amount of tumour slices in our dataset was significantly lower than the quantity of normal slices. To overcome this, oversampling was used and the data augmentation was done. The tumour slices were rotated randomly by 5-10 degrees to obtain new slices and this helped us to train our model more effectively.

The images in the dataset have manual markings of the tumours identified in the MRI scans and these manual markings are used to check the precision and accuracy of our model.

\section{U-Net Description}

U-Net, advanced from the conventional convolutional neural system, was first structured and applied in 2015 to process biomedical pictures. As a general convolutional neural system concentrates its undertaking on picture arrangement, where information is a picture and yield is one mark, 
however in biomedical cases, it requires us not exclusively to recognize whether there is a malady, yet additionally to restrict the region of variation from the norm.

U-Net is devoted to taking care of this issue. The explanation it can limit and recognize fringes is by doing grouping on each pixel, so the info and yield share a similar size.

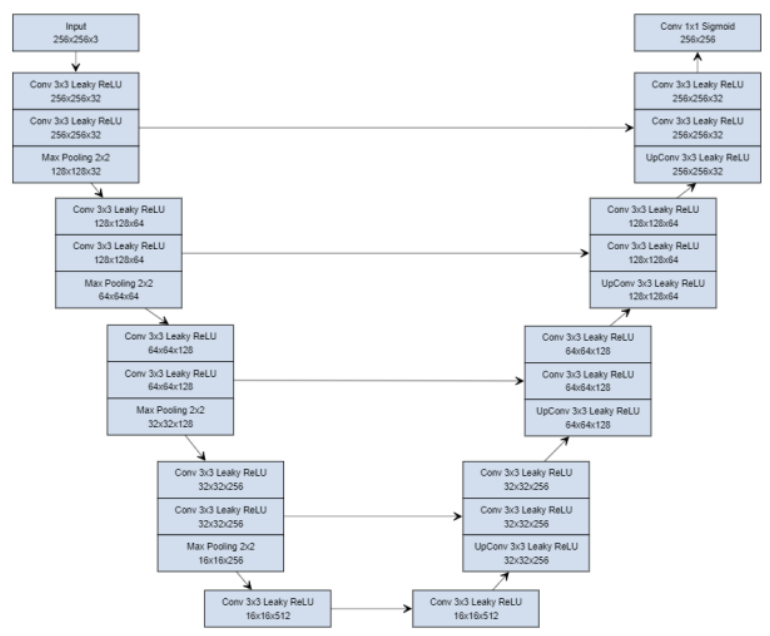

Figure 6: The U-Net Architecture of our model

\section{Dice Loss Coefficient}

Dice loss was developed in the 1940s to compare the amount of resemblance shown between two or more sample images. It was developed using the SorensonDice coefficient [8]. Millitari brought this measurement statistic to the computer vision community in order to develop a model to segment 3 dimensional medical images.

$$
\mathrm{D}=\frac{2 \sum_{\mathrm{i}}^{\mathrm{N}} \text { aibi }}{\sum_{\mathrm{i}^{\mathrm{N}}}^{\mathrm{N}} \mathrm{ai}^{2}+\sum_{\mathrm{i}^{\mathrm{N}}}^{\mathrm{N}} \mathrm{bi}^{2}}
$$

Here ai and bi are the values of the pixels of the image and the truth value of the pixels. When a boundary is detected the corresponding values of ai and bi become either 0 or 1 signifying that a pixel is at the boundary or not. In this way, the divisor is the sum of complete limit pixels of both forecast and ground truth, and the dividend is the whole of accurately anticipated limit pixels in the wake of the fact that the entirety increases just when ai and bi coordinate (both of significant worth 1).

\section{Results and Discussion}

Out of all the 110 images dataset, the deep learning algorithm gave a mean Dice Coefficient of $85 \%$ and a median Dice Coefficient of $87 \%$. Network layers in U-Net have max pooling enabling us to work on large amounts of data and therefore our segmentation techniques is less effective for high curvature and the predicted masks are more smooth than the manual masks. We also tried to input noise into the images so as to prevent over fitting of our model which would have happened given the high class imbalance in our dataset.

\begin{tabular}{|l|l|l|}
\hline Noise Inserted & Mean dice coefficient & Median dice Coefficient \\
\hline $0 \%$ & $85 \%$ & $87 \%$ \\
\hline $10 \%$ & $83 \%$ & $87 \%$ \\
\hline
\end{tabular}

Figure 7: Loss vs dice coefficient in our model.

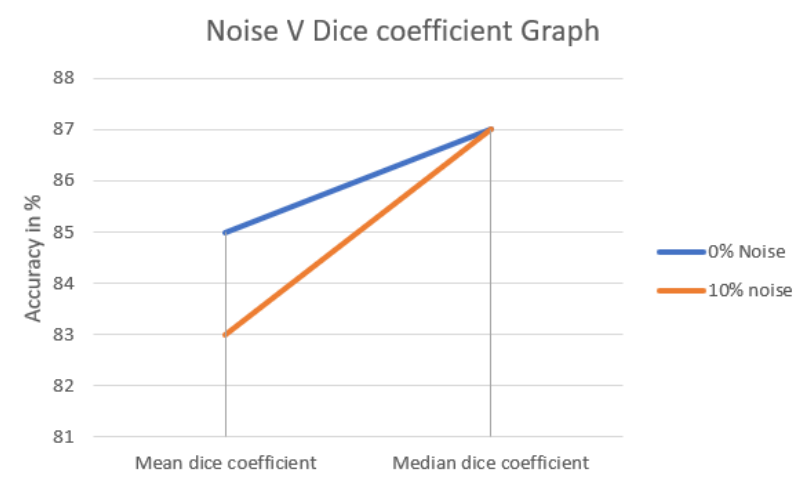

Figure 8: Graphical representation of noise vs dice coefficient.

Here, we see that when noise is inserted into the dataset to prevent overfitting of our model the mean dice coefficient is reduced from $85 \%$ to $83 \%$ but the median dice coefficient remains the same at $87 \%$.

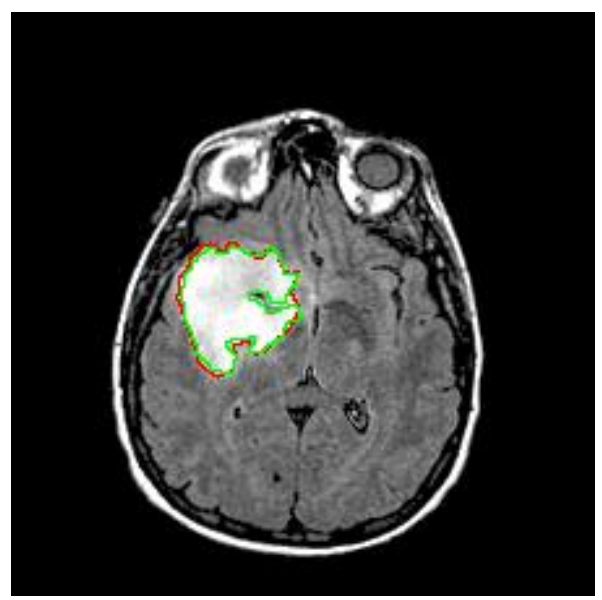

Figure 9: Sample output 


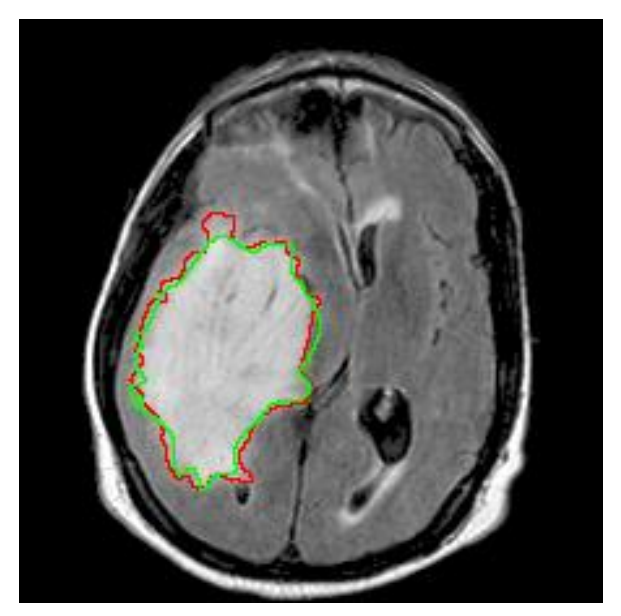

Figure 10: Sample output

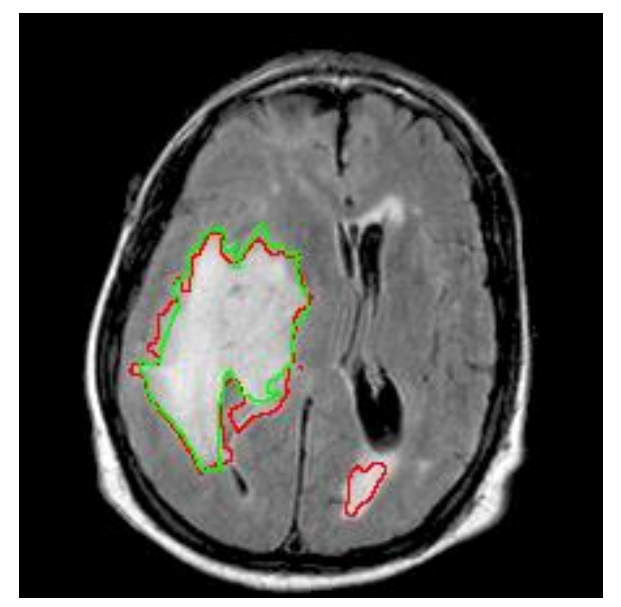

Figure 11: Sample Output

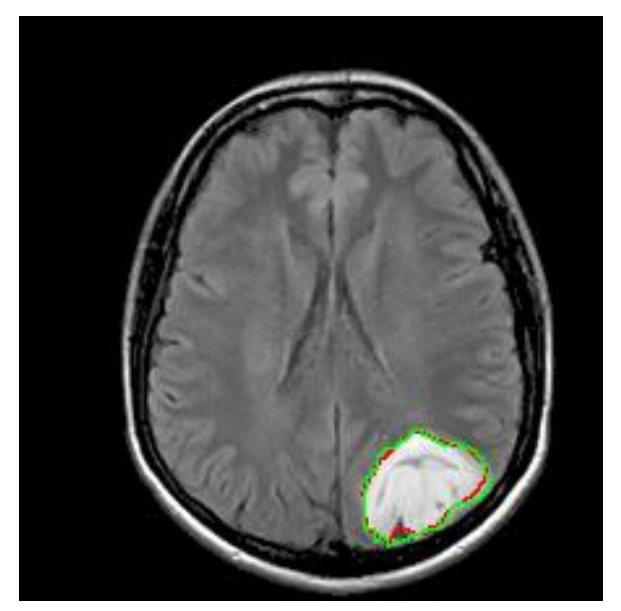

Figure 12: Sample Output

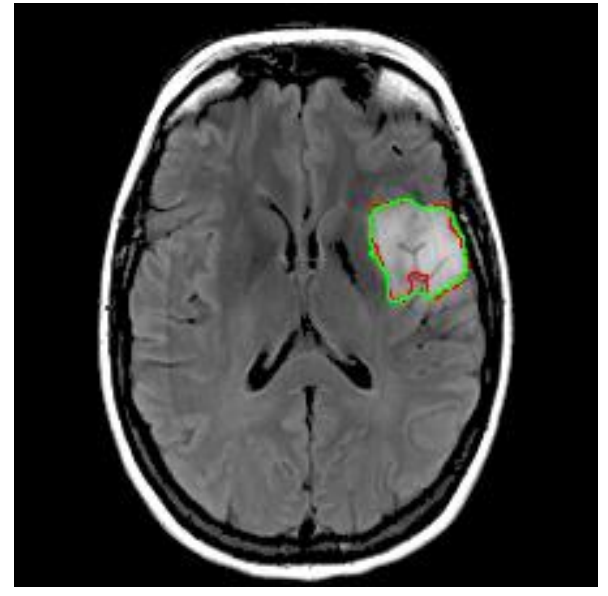

Figure 13: Sample Output

Here in the figures 9-13, the red outline is the predicted tumour region where as the green outline represent the manually segmented tumour region.

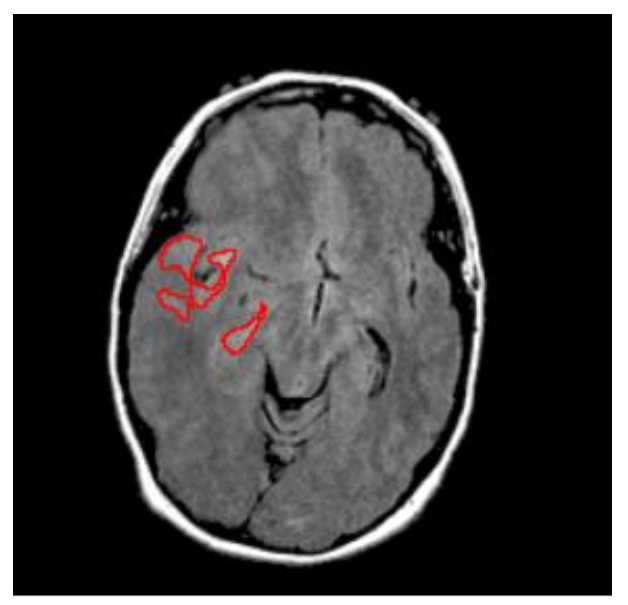

Figure 14 - We see due to lack of extensive training cases the Figure 14 incorrectly masks the tumour but in reality there is no tumour at all.

Comparing our results with previously conducted researches on the LGG MRI[9] dataset we see that, our model able to handle a very large amount of images on our given systems. The noise inserted into our image also helps increase overall accuracy for new MRI images by preventing overfitting of our model on the given dataset itself.

Also, our model achieved a dice coefficient of $85 \%$ which is better than the dice coefficient of $82 \%$ by using ReLU activation function[9] in the neural network layers. 


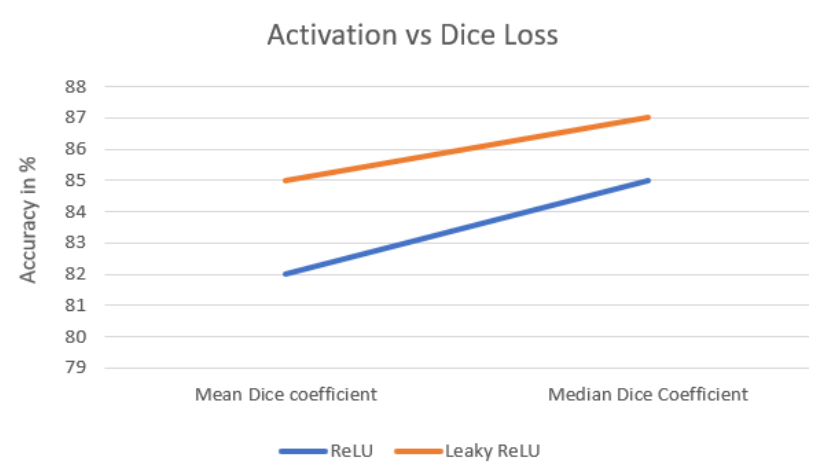

Due to limited number of images (110 patients) our model gave incorrect output for Figure 14. This short coming can be removed by:

Training our model on a larger dataset

Identifying various features like relation between boundary tumour tissues and normal tissues so it can be incorporated into our model to increase its accuracy.

Compared to Dong et al., [5], Cui et al., [6] which were trained and tested on BRATS dataset, our model gave better segmentation results for core tumour segmentation. However our model was ineffective in segmenting very complex and distributed tumours as the training dataset was very less.

\section{Conclusion}

In this study, we were able to develop a model segmenting the tumours images. Further if data regarding the properties of the tumours is available then a model can be developed to identify the weights of these properties on the nature of the tumour. For example, if data about different clusters inside the brain is made available then we can carefully identify the properties of these clusters like RNAclusters, etc and use this data to identify the properties of the tumours. This analysis coupled with tumour segmentation can be very useful for the doctors and can help medical professional to carry out accurate and verified analysis and segmentation of the MRI scans of the patients under their supervision. Thus, the impact of AI in medical field increases everyday

As brain tumour dataset contain sensitive patient information it is very difficult to acquire very large dataset.

So, our deep learning model has proven successful in segmenting the tumour cells apart from the healthy cells to a satisfactory level of precision. Also, on our website anyone can upload their MRI images and check if they have a tumour or not. However due to lack of more data the model sometimes marks healthy cells as tumours as well. This can be avoided when the data set is bigger and the model can learn correctly the features and boundaries of a tumour cell.

I would like to thank The Cancer Imaging Archive (TCIA) for compiling and providing this dataset.

\section{References}

1. Hanif F, Muzaffar K, Perveen K, Malhi SM, Simjee ShU. Glioblastoma Multiforme: A Review of its Epidemiology and Pathogenesis through Clinical Presentation and Treatment. Asian Pac J Cancer Prev. 2017;18(1):3-9. Published 2017 Jan 1. doi:10.22034/APJCP.2017.18.1.3

2. Havaei, Mohammad, et al. "Brain tumor segmentation with deep neural networks," Medical image analysis, vol. 35, pp. 18-31, 2017.

3. Parihar, Anil Singh. "A study on brain tumor segmentation using convolution neural network," Inventive Computing and Informatics (ICICI), International Conference on. IEEE, 2017.

4. Dvorak, Pavel \& Menze, B.. (2015). Structured prediction with convolutional neural networks for multimodal brain tumor segmentation. 13-24.

5. Dong H, Yang G, Liu F, Mo Y, Guo Y. Automatic brain tumor detection and segmentation using $U$-net based fully convolutional networks. MIUA. 2017;3:1-12.

6. Cui S, Mao L, Jiang J, Liu C, Xiong S. Automatic semantic segmentation of brain gliomas from MRI images using a deep cascaded neural network. J Healthcare Engineering. 2018:1-14.28.

7. Chinmayi P, Agilandeeswari L, Prabu Kumar M, Muralibabu K. An efficient deep learning neural network based brain tumor detection system. Int Pure Appl Mathemat. 2017;117:151-60.

8. I. Binanto et al., "Comparison of Similarity Coefficients on Morphological Rodent Tuber," 2018 Indonesian Association for Pattern Recognition International Conference (INAPR), Jakarta, Indonesia, 2018, pp. 104-107, doi: 10.1109/INAPR.2018.8627050. 
9. Buda, Mateusz \& Saha, Ashirbani \& Mazurowski, Maciej. (2019). Association of genomic subtypes of lower-grade gliomas with shape features automatically extracted by a deep learning algorithm. Computers in Biology and Medicine. 109. 10.1016/j.compbiomed.2019.05.002.

\section{Creative Commons Attribution License 4.0} (Attribution 4.0 International, CC BY 4.0)

This article is published under the terms of the Creative Commons Attribution License 4.0

https://creativecommons.org/licenses/by/4.0/deed.en_US 